\title{
The microbiota in inflammatory bowel disease: current and therapeutic insights
}

\author{
This article was published in the following Dove Press journal: \\ Journal of Inflammation Research \\ 10 June 2017 \\ Number of times this article has been viewed
}

\author{
Erin R Lane' \\ Timothy L Zisman ${ }^{2}$ \\ David L Suskind' \\ 'Division of Gastroenterology \\ and Hepatology, Seattle \\ Children's Hospital, ${ }^{2}$ Division of \\ Gastroenterology, University of \\ Washington, Seattle, WA, USA
}

Correspondence: Timothy L Zisman University of Washington Medical

Center, 1959 NE Pacific St., Box 356424,

Seattle, WA 98195, USA

Tel +l 2066857I74

Fax +I 2066858684

Email tzisman@uw.edu
Abstract: Inflammatory bowel disease is a heterogeneous group of chronic disorders that result from the interaction of the intestinal immune system with the gut microbiome. Until recently, most investigative efforts and therapeutic breakthroughs were centered on understanding and manipulating the altered mucosal immune response that characterizes these diseases. However, more recent studies have highlighted the important role of environmental factors, and in particular the microbiota, in disease onset and disease exacerbation. Advances in genomic sequencing technology and bioinformatics have facilitated an explosion of investigative inquiries into the composition and function of the intestinal microbiome in health and disease and have advanced our understanding of the interplay between the gut microbiota and the host immune system. The gut microbiome is dynamic and changes with age and in response to diet, antibiotics and other environmental factors, and these alterations in the microbiome contribute to disease onset and exacerbation. Strategies to manipulate the microbiome through diet, probiotics, antibiotics or fecal microbiota transplantation may potentially be used therapeutically to influence modulate disease activity. This review will characterize the factors involved in the development of the intestinal microbiome and will describe the typical alterations in the microbiota that are characteristic of inflammatory bowel disease. Additionally, this manuscript will summarize the early but promising literature on the role of the gut microbiota in the pathogenesis of inflammatory bowel disease with implications for utilizing this data for diagnostic or therapeutic application in the clinical management of patients with these diseases.

Keywords: Crohn's disease, ulcerative colitis, microbiome, fecal microbiota transplantation, enteral nutrition, specific carbohydrate diet

\section{Introduction}

Inflammatory bowel disease (IBD) represents a heterogeneous group of chronic immune-mediated inflammatory diseases affecting the gastrointestinal tract. There are two primary phenotypes of IBD, ulcerative colitis (UC) and Crohn's disease (CD). $\mathrm{UC}$ is characterized by chronic contiguous and circumferential mucosal inflammation extending proximally from the rectum, but is isolated to the colon. In comparison, the stereotypical inflammation seen in CD is patchy and transmural, and may affect any part of the gastrointestinal tract. Although the etiology of IBD is incompletely understood, recent studies support the hypothesis that IBD results from a complex interplay of genetics, immune dysregulation and environmental triggers that may exert their effect through alterations of the intestinal microbiota.

Currently, IBD affects an estimated 1.4 million individuals in the USA, with a significant increase in incidence over the past decades. This has spurred the hypothesis 
that environmental factors play a critical role in the pathogenesis of IBD. ${ }^{1,2}$ Further evidence of environmental impact on disease development is seen in the discordant incidence of IBD in industrialized compared with non-industrialized countries, as well as the rising incidence of IBD in countries that are undergoing demographic and economic development. ${ }^{3,4}$ Similarly, children emigrating from countries with low IBD prevalence to countries with high IBD prevalence assume the same risk of developing IBD as their peers residing in high IBD prevalence areas for many generations. ${ }^{5}$ While genes play a clear role in the pathogenesis of IBD, with over 200 genes predisposing to IBD discovered so far, the critical role of environment is further highlighted by monozygotic twin studies, where concordance of IBD among monozygotic twin pairs is $<50 \%$ as well as the fact that there is incomplete penetrance of gene abnormalities predisposing to IBD in the general population. ${ }^{6-8}$

Current theories of IBD pathogenesis postulate that pathologic alterations in the intestinal microbiome trigger an aberrant mucosal immune response in genetically predisposed individuals, leading to the development of chronic intestinal inflammation. These pathologic alterations in gut microbial composition seen in IBD are referred to as intestinal "dysbiosis." Research suggests that perturbations in the gut microbiome are an essential factor triggering inflammation in IBD rather than merely a consequence of the chronic inflammation. ${ }^{9}$ Abundant evidence supports the integral role the intestinal microbiome plays in the pathogenesis of IBD, including: 1) a characteristic dysbiosis is often observed in individuals with $\mathrm{CD}$, UC and pouchitis, ${ }^{10-16}$ 2) fecal stream diversion improves disease activity in $\mathrm{CD}$, whereas reinfusion of fecal contents results in recurrent inflammation, ${ }^{17}$ 3 ) the majority of IBD susceptibility genetic polymorphisms are associated with host mucosal barrier function and are involved in host-microbiome interactions, ${ }^{18-23} 4$ ) antibiotics and probiotics have been shown to be effective for induction or maintenance of remission in IBD ${ }^{24-26} 5$ ) depletion of commensal microbes can result in impaired mucosal healing, chronic mucosal inflammation and colitis, ${ }^{27}$ and 6) germ-free animals do not develop colitis without introduction of fecal bacteria to induce inflammation. ${ }^{28}$

While advances in bioinformatics, genomics and experimental models of IBD have identified how environmental factors such as age, diet and antibiotic exposure contribute to the development of dysbiosis and aberrant gut microbial-host immunologic interactions, many questions remain. The following review aims to: 1 ) describe factors in the development of the intestinal microbiome, 2) define features of intestinal dysbiosis in IBD, and, 3) explore how current knowledge may lead to the development of therapeutic interventions by harnessing the microbiome in the treatment of IBD.

\section{The microbiome in health: factors that influence development, alteration and maintenance of structure and function}

The gut microbiota is the largest and most diverse community of microbes in the human body. The intestinal microbiota, or microorganism population of the intestine, constitutes only a fraction of the complexity of the intestinal microbiome, which includes diverse array of microbial genes and gene products of the microbiota. During times of good health, the intestinal microbiome acts symbiotically to produce vitamins, repress expansion of pathologic organisms and facilitate digestion of dietary substrates, all the while in constant contact with the host immune system. ${ }^{29}$ Additionally, the microbiome contributes to gut epithelial cell renewal and enteric immune system development. This diverse community of bacteria, fungi, bacteriophages, and archaea exist in colonies of varying density throughout the gastrointestinal tract, with the highest microbial density reaching $10^{12}$ cells/g of luminal contents in the colon. ${ }^{30}$ Attempts have been made to delineate what constitutes a "healthy" microbial composition of the intestinal microbiome, with varying and somewhat disparate results. The difficulty in defining a "healthy" microbiome is based upon the complexity and variations found in the fecal microbiome, with more than 1000 potential bacterial species able to colonize the human intestines. Despite the large variation found in an individual's microbiome, the majority of species $(>90 \%)$ belong to the Bacteroidetes and Firmicutes phyla. ${ }^{31,32}$

The composition of the gut microbiota changes over time. An individual's intestinal microbiome is more malleable in infancy and early childhood, assumes more stability and similarity to the general population in adulthood and subsequently loses diversity in the elderly. ${ }^{12,33}$ During infancy, the gut microbiome is typically minimally complex and is influenced by birth route and diet. ${ }^{34-36}$ Method of delivery (cesarean section versus vaginal delivery) differentially affects the neonatal intestinal microbiota composition, with vaginal and fecal flora colonizing the newborn gut if born vaginally and typical skin flora if born via cesarean section. ${ }^{37}$ A Danish cohort study of infants born between 1973 and 2008 demonstrated that cesarean sections were associated with a moderately increased risk of IBD; however, this finding was recently disputed by Bernstein et al, who observed 
that patients with IBD were not more likely to have been born via cesarean section than controls or their siblings without IBD. ${ }^{38,39}$

Infant diet also impacts the composition of the intestinal microbiome. Exclusively breastfed infants have been found to have increased numbers of taxa from Actinobacteria whereas formula-fed infants have higher levels of $\gamma$-Proteobacteria. ${ }^{40,41}$ Additionally, formula-fed infants demonstrate more "adult"like patterns of intestinal microbiota. Interestingly, intestinal microbiota of breast milk-fed infants are significantly less diverse than formula-fed infants, but their microbial genes demonstrated more robust interactions with host immune system, metabolism and biosynthesis. ${ }^{42}$ There is a reduced risk of development of IBD among infants who were breastfed. ${ }^{43}$ The cessation of breastfeeding and associated reduction in passage of maternal IgA induces changes in the microbiome characteristic of an adult microbiome, including increased prominence of Firmicutes and Bateriodetes; however, peak microbial diversity and microbiome stability is often not reached until adulthood. ${ }^{44-46}$

During childhood and adulthood, diet appears to have a major influence on microbial composition and function. ${ }^{47} \mathrm{~A}$ controlled study examining the effects of diet on the microbiome demonstrated that the composition of gut microbiota is changed dramatically by increases in dietary fat and decreases in dietary fiber compared with low-fat/highfiber diets. ${ }^{48}$ African children on high-fiber vegetarian diets demonstrate different gut microbiomes than their European peers, whose diets are typified by high sugar, fat and animal protein. ${ }^{49}$ Diets rich in animal fats have also been shown to favor increased density of bile-tolerant organisms, including Bilophila wadsworthia.$^{47}$ Interleukin (IL)-10 knockout mice fed diets rich in milk-derived saturated fat favored colonization with Proteobacteria (specifically B. wadsworthia), compared with diets high in polyunsaturated fat. Interestingly, these IL-10 knockout mice fed diets high in saturated milk fat demonstrated increased incidence and severity of spontaneous colitis, providing a potential mechanistic link between the observed association between the proliferation of Westernized diets and increased incidence of IBD globally. ${ }^{50}$

Similarly, low vitamin $\mathrm{D}$ has been identified as a potential risk factor for the development of IBD. ${ }^{51}$ Vitamin D deficiency may contribute to intestinal inflammation through multiple effects and alters the gut microbiome. ${ }^{52}$ Alternatively, supplementation with vitamin $\mathrm{D}$ has been shown to alter the intestinal microbiome by increasing alpha diversity (species richness). ${ }^{53}$ While abundant data support dietary influences as having a dramatic, either pro-inflammatory or anti-inflammatory effect on the intestinal microbiome, further research is required to delineate the mechanism by which specific dietary alterations are useful in the treatment of IBD.

\section{Alterations in intestinal microbiome implicated in the development of IBD}

While IBD is clearly associated with intestinal dysbiosis, no single microbe or microbial milieu has been proven causal. Intestinal dysbiosis may contribute to the pathogenesis of IBD by loss of "health-promoting" or potential gain of "pathobionts." Pathobionts are distinguishable from bacterial pathogens in that they only become pathologic in the setting of a specific environmental stimulus in genetically susceptible individuals. With recent advances in genetic sequencing and functional microbial analysis, many studies have been able to identify intestinal dysbioses that are present in patients with IBD. While there are somewhat disparate results, common themes among studies support the finding of a generalized reduction in biodiversity (alpha diversity) as well as decreased representation of several specific taxa, including Firmicutes and Bacteroidetes, among individuals with IBD. ${ }^{10-16}$ Additionally, specific taxonomic shifts have been associated with IBD, including a relative increase in the abundance of Enterobacteriaceae, including Escherichia coli and Fusobacterium. ${ }^{54-56}$ Newly diagnosed, treatment-naïve patients provide an ideal human study population in which to observe the potentially pathologic intestinal dysbioses that occur in IBD. Interestingly, in a large cohort of newly diagnosed, treatment-naïve children with $\mathrm{CD}$, ileal and rectal biopsy samples demonstrated an increased abundance of Enterobacteriaceae, Pasteurellaceae, Veillonellaceae, and Fusobacteriaceae and decreased abundance of Bacteroidales and Clostridia. ${ }^{13}$ Fite et al demonstrated that not only do mucosa-associated microbes differ between those with active colonic inflammation (UC) and those with normal mucosa, there appears to be longitudinal variation in mucosal bacterial populations in UC that is associated with disease severity. ${ }^{57}$ Moreover, fecal microbiota composition in active $\mathrm{UC}$ is consistent across geographic location, age and gender, and IBD-associated microbiota appears to be stable during remission among those with UC. ${ }^{58}$

Although fewer studies have examined the role of fungi specifically in the propagation of inflammation in IBD, fungi are a ubiquitous component of the intestinal microbiome. In $\mathrm{CD}$, intestinal fungal communities have increased diversity in colonic and ileal biopsy samples compared with healthy controls. ${ }^{59,60}$ In pediatric patients with $\mathrm{CD}$, five fungal taxa were found to be associated with $\mathrm{CD}$, including: Saccharomyces 
cerevisiae, Calvispora lusitaniae, Cyberlindnera jadinii, Candida albicans, and Kluyveromyces marxianus. ${ }^{61}$ In addition, fungal microbial components are utilized in the diagnosis and prognosis of CD; specifically anti-saccharomyces cerevisiae antibodies (ASCA), which react with a yeast cell wall polysaccharide, serve as a serological marker for ileal CD. ${ }^{1}$ A recent study demonstrated increased abundance of Candida tropicalis in CD patients compared with their unaffected relatives, and this correlated with ASCA titers and abundance of Serratia marcescens and E. coli in biofilms. ${ }^{62}$ Like fungi, bacteriophages are an understudied population of the intestinal microbiome. Colonic mucosal biopsy samples from patients with $\mathrm{CD}$ had significantly more bacteriophage than healthy controls. ${ }^{63,64}$ However, given the relative paucity of data examining the functional role of fungi, viruses and archaea in the intestinal microbiome, further studies are required to develop a deeper understanding of how changes in these populations may result in IBD.

Interestingly, studies have demonstrated a reduced diversity of intestinal microbiota in inflamed versus normal intestinal mucosa within the same patient, suggesting that host immune factors play a role in maintaining or reacting to mucosal inflammation. ${ }^{65}$ It has long been suspected that an individual's genotype contributes to overall susceptibility in developing IBD. Studies evaluating specific genetic variants have highlighted the interplay between the gut immune system and intestinal microbiota. The first gene identified to confer susceptibility in developing CD was nucleotide-binding oligomerization domain containing 2 (NOD2) ${ }^{66}$ Under normal circumstances, NOD2 is primarily expressed in Paneth cells, the role of which in the intestinal mucosa is to produce antimicrobial defensins. The product of NOD2 stimulates host immune response upon recognition of microbial cell wall components. Patients with NOD2 mutations have been found to have decreased IL-10, an important anti-inflammatory cytokine. Additionally, those with NOD2 mutations have been found to harbor increased intestinal mucosal adherent bacteria. ${ }^{10,67}$ In mice, intestinal microbiota affects expression of NOD2. ${ }^{68}$ NOD2-deficient mice demonstrate significant increases in Bacteroides, Firmicutes, and Bacilli in their terminal ileum, and decreased ability to clear pathogenic Helicobacter hepaticus. ${ }^{68}$ Patients with NOD2 risk alleles have decreased clostridium groups XIVa and IV and increased Actinobacteria, Proteobacteria and Enterobacteriaceae. ${ }^{10}$ Since the initial identification of NOD2 and associated risk alleles, over 160 genetic loci have been identified to modulate the risk of IBD. ${ }^{6,19,69}$ Furthermore, genetic analyses have linked specific loci associated with a dysregulated intestinal immune response to commensal microbes with the development of IBD. ${ }^{6}$

Analogous to the dynamic relationship between mucosal bacterial milieu and the host immune system, fungal microbiome communities interact with host immune cells via the innate immune receptor, Dectin- $1 .{ }^{70}$ Dectin-1 recognizes fungal cell wall glucans and activates intracellular signals through the CARD9 receptor, leading to production of inflammatory cytokines and subsequent induction of Th17 responses. ${ }^{70}$ Variants in CARD9 and Decin-1 are associated with $\mathrm{CD}$ and UC, specifically, mutations in Dectin-1 $(C L E C 7 A)$ are associated with medically refractory UC. ${ }^{69,70}$

\section{Environmental factors affecting the intestinal microbiome and the development of IBD}

While antibiotics may have a therapeutic role in established IBD, several studies have shown that antibiotic exposure prior to the development of IBD is associated with incident IBD. In a retrospective cohort of Danish children, a greater relative risk of developing IBD was observed among children exposed to antibiotics. ${ }^{71}$ Likewise, a Finnish study demonstrated the risk of pediatric-onset $C D$ increased with the number of purchased courses of antibiotics from birth. ${ }^{72} \mathrm{~A}$ large retrospective cohort study from the UK demonstrated that exposure to antibiotics, particularly anaerobic antibiotics, during childhood was associated with a relative rate of increase in the development of IBD by $84 \% .^{73}$ In a recent meta-analysis, exposure to antibiotics during childhood was shown to be associated with increased risk of CD but not UC. ${ }^{74}$ Together, these findings suggest that early life and repeated exposures of antibiotics may result in sustained, potentially detrimental effects on the intestinal microbiome and could contribute to the pathogenesis of IBD.

As previously mentioned, diet has a significant effect on the fecal microbiome. With this in mind, several large longitudinal studies have demonstrated an association between reduced risk of IBD and a diet high in fruits and vegetables, and an elevated risk of IBD in those who consumed diets rich in animal fats and refined sugars. ${ }^{4}$ In addition, Western diets high in fat have been demonstrated to increase risk of IBD; specifically, consumption of a high ratio of omega-6 fatty acids (pro-inflammatory) to omega-3 fatty acids (antiinflammatory) has been associated with an increased incidence of UC. ${ }^{75,76}$ In mice, a diet high in n-6 polyunsaturated fatty acids exacerbated colitis and resulted in enrichment of the intestinal microbiome with pro-inflammatory Enterobacteriaceae and Clostridia spp. ${ }^{77}$ 


\section{The intestinal microbiome as a diagnostic and therapeutic tool}

While earlier studies focused on species characterization of the intestinal microbiome in health versus disease, advances in metagenomics and metabolomics have introduced the importance of understanding the functional properties of the intestinal microbiome in IBD. Specifically, such functional studies have highlighted differences in: carbohydrate and lipid transport/metabolism, cell wall degradation, formation of exotoxins, and microbial adherence and invasion in IBD versus in health. ${ }^{1}$ In defining the functional role of the intestinal microbial community in disease and health, there is a directive to harness this knowledge to restore health to a diseased microbiome through diet, prebiotics, probiotics, antibiotics and/or fecal microbiota transplantation (FMT).

Diet has an immense impact on gut microbiome development and diversity, and has been well studied as a potential therapy for IBD. Perhaps the largest body of literature on use of diet as a targeted IBD therapy evaluates the efficacy and microbial changes associated with exclusive enteral nutrition (EEN). EEN is a complete exclusion diet, where patients receive $100 \%$ of their daily calorie intake from formula rather than table foods. EEN may be completed with intact protein formula, semi-elemental or elemental formula. Studies employing metagenomics have demonstrated alterations in the intestinal microbiome before and after treatment with EEN. ${ }^{61}$ EEN has been shown to induce alterations in the microbiome as early as 1 week after initiation. Interestingly, the intestinal microbiome in these individuals did not resemble that of healthy individuals. Similar results were demonstrated by Gerasimidis et al in a prospective, casecontrol study evaluating changes in fecal microbial diversity and metabolic activity of 15 children with CD treated with EEN. ${ }^{78}$ Soon after initiation, and throughout treatment with EEN, fecal microbiota diversity decreased, as did concentrations of previously identified commensal microbes. These observations correlated with an improvement in clinical disease activity and reduction in inflammatory markers. ${ }^{78,79}$ Interestingly, these differences in microbiome were more pronounced in study subjects who responded to EEN compared with those who did not, potentially identifying biomarkers of disease phenotype and aiding future selection of individual patients who are more likely to respond to particular therapies. Analogous changes to the structure of the intestinal microbiome were not demonstrated in children on partial enteral nutrition (PEN; a diet composed of partial formula and table foods) despite receiving a similar volume of enteral formula, suggesting that the changes to the microbiome seen with EEN may stem from exclusion of table foods. ${ }^{80}$

EEN in CD has been shown to improve clinical symptoms, mucosal healing and nutritional status in children, with clinical remission rates equivalent to systemic corticosteroids ${ }^{81}$ In a randomized controlled trial evaluating the efficacy of steroids versus EEN for treatment of CD, both treatments equally improved clinical symptoms as well as serum inflammatory markers, but EEN resulted in significantly better mucosal healing. ${ }^{81}$ Similar clinical results have not been reproduced in adults, as EEN resulted in lower rates of clinical response than steroids, although this may be a question of compliance versus efficacy. ${ }^{80}$

While EEN for induction of remission is clearly beneficial for children with $\mathrm{CD}$, it is often less feasible for long-term maintenance of remission. When combined with a regular diet, even $50 \%$ of calories from EEN reduce the rate of $\mathrm{CD}$ relapse by $50 \% .^{82}$ However, effectiveness at clinical and mucosal remission is greatest when $100 \% \mathrm{EEN}$ is employed, rather than PEN. ${ }^{83,84}$ Given that many patients feel EEN to be prohibitively restrictive, there have been efforts to identify whole food diets that may confer similar therapeutic benefit.

The specific carbohydrate diet (SCD) is one such exclusion diet that appears to have a positive effect in IBD. The SCD diet was developed by Dr Sydney Haas, a pediatrician in the 1930s who developed the diet as a treatment for celiac disease. This diet removes all grains, including gluten. It was the primary treatment for celiac disease prior to the discovery of gluten as the offending antigen. The SCD diet was then popularized by Elaine Gottschall as treatment for IBD after her daughter's UC was successfully treated with the diet. The diet also removes sweeteners except for honey, most processed foods and all milk products, except for hard cheeses and yogurt fermented $>24$ hours. In an online survey of 417 IBD patients ( $47 \% \mathrm{CD}$ and $43 \% \mathrm{UC}$ ) who used the $\mathrm{SCD}$ as treatment, $33 \%$ reported remission at 2 months after initiation of the SCD, and $42 \%$ at both 6 and 12 months. For those reporting clinical remission, $13 \%$ reported time to achieve remission of $<2$ weeks, $17 \%$ reported 2 weeks to a month, 36\% reported 1-3 months, and 34\% reported $>3$ months. For individuals who reported reaching remission, $47 \%$ of individuals reported associated improvement in abnormal laboratory values ${ }^{85}$ Suskind et al reported clinical remission in seven children with active $\mathrm{CD}$ after initiation of the SCD diet without use of concomitant treatment. ${ }^{86} \mathrm{In}$ addition to clinical remission, these children demonstrated normalization/improvement inflammatory markers, including fecal calprotectin. Cohen et al reported clinical and mucosal 
improvements, as documented with capsule endoscopy in seven children with CD who used SCD for 52 weeks. ${ }^{87}$ In addition to the aforementioned studies, case series including over 75 patients have shown clinical and laboratory improvement in both CD and UC patients on SCD. ${ }^{88,89}$

In a recent prospective multicenter study of SCD in pediatric patients with mild-to-moderate $\mathrm{CD}$ or $\mathrm{UC}, 12$ pediatric patients (aged 10-17 years) were followed for 12 weeks. Dietary therapy was associated with clinical remission in eight patients and was ineffective for two patients, while two individuals were unable to maintain the diet. At 12 weeks, the mean C-reactive protein decreased from $24.1 \pm 22.3 \mathrm{mg} / \mathrm{L}$ to $7.1 \pm 0.4 \mathrm{mg} / \mathrm{L}$ in the Seattle cohort $(\mathrm{nL}<8.0 \mathrm{mg} / \mathrm{L})$ and from $20.7 \pm 10.9 \mathrm{mg} / \mathrm{L}$ to $4.8 \pm 4.5 \mathrm{mg} / \mathrm{L}$ in the Atlanta cohort $(\mathrm{nL}<4.9 \mathrm{mg} / \mathrm{L})$. Concomitant with clinical and laboratory improvements, significant changes in microbial composition occurred with the dietary change. ${ }^{90}$

Other exclusion diets have been used for reduction of inflammation and symptom improvement in IBD. The Crohn's Disease Exclusion Diet (CDED), designed and evaluated by Dr Arie Levine, is based on the hypothesis that the efficacy of EEN is dependent on exclusion of dietary components that may potentially influence intestinal permeability or promotion of a pro-inflammatory microbiome. Foods specifically excluded from the diet include: gluten, dairy, gluten-free baked goods, animal fat, processed meats, products containing emulsifiers and all canned or processed foods. In a prospective cohort of pediatric and adult patients with mild-to-moderate CD treated with 50\% PEN and the CDED, Sigall-Boneh et al, showed this exclusion diet was successful in achieving induction of clinical remission, including a reduction in inflammatory markers. ${ }^{91}$ Additionally, a small subgroup of seven patients treated with the CDED alone also achieved clinical remission. ${ }^{91}$ Similarly, Riordan et al demonstrated that in adults with active $\mathrm{CD}$, after induction of elemental diet, those who excluded trigger foods had prolonged clinical remission and improvement in serum inflammatory markers compared with those who consumed a regular diet. ${ }^{92}$ Foods most frequently excluded owing to "intolerances" included: cereals, dairy products and yeast. Similarly, Rajendran et al found that IgG4-targeted exclusion diets for adults with active $\mathrm{CD}$ resulted in symptomatic improvement and reduction in serum inflammatory markers. ${ }^{93}$ In a small, prospective controlled study of adults with CD in clinical remission, Chiba et al demonstrated that a lacto-ovo-vegetarian diet resulted in reduction in relapse and prolongation of time to relapse versus a standard omnivorous diet. ${ }^{94}$ While these exclusion diets hold promise for induction or maintenance of remission in $\mathrm{CD}$, further research is necessary to characterize their effects on mucosal healing and alterations in microbiome.

Other diets have not been as well studied in IBD; however, low-lactose diets have been shown to reduce clinical symptoms of IBD. Similarly, patients with IBD report symptom improvement on low FODMAP (Low Fermentable oligosaccharides, disaccharides, monosaccharides, and polyols) diet, but there is paucity of data that supports a reduction in intestinal mucosal inflammation. ${ }^{95,96}$ Low FODMAP diets have been found to reduce potentially favorable bacterial species within the colon, particularly Faecalibacterium prausnitzii and reduced production of butyrate. ${ }^{97}$ However, these alterations in the microbiome have unclear clinical consequences. Other restriction diets such as the vegan and vegetarian diets are popular among some patient populations. Zimmer et al demonstrated alterations in the ratios of Bacteroides spp., Bifidobacterium supp., and Enterobacteriaceae in patients adherent to vegan and vegetarian diets. ${ }^{98}$ Further studies are required before these restriction diets can be recommended for the treatment of IBD.

The mechanism of action in reduction of clinical symptoms and intestinal inflammation with EEN and exclusionary diets remains unclear. Restriction diets may act through exclusion of certain substrates that modulate the intestinal microbiome, or may be related to the exclusion of food additives, including emulsifiers that may modulate the intestinal microbiome and/or directly affect the immune system through alterations in the mucus barrier as well as the epithelial lining. ${ }^{80}$ Murine models of colitis (IL-10 knockout mice) fed common commercial food emulsifiers, carboxymethylcellulose and polysorbate- 80 demonstrated thinning of the colonic mucosal layer, invasion of bacteria into the lamina propria, altered microbiome and worsening colitis. ${ }^{99}$ Nickerson et al has demonstrated parallels between the increasing dietary prevalence of maltodextrin (MDX), a common starch-based food additive, and rising incidence of CD. Through a series of experiments to uncover potential mechanisms, Nickerson et al demonstrated that exposure to MDX enhances mucosal adhesion and biofilm formation by $E$. coli, including adherent-invasive $E$, coli, and increases viability of intracellular Salmonella in mucosal macrophages and epithelial cells. Using this evidence, they hypothesize that MDX may contribute to the development of IBD by priming the intestinal mucosal to be more sensitive to epithelial damage due to a reduction in epithelial antimicrobial defense mechanisms. ${ }^{100}$ In sum, while there are promising preliminary data supporting the use of exclusion diets in the treatment of IBD, additional studies are required to draw 
definitive conclusions regarding how exclusion diets influence the intestinal microbiome composition and function and how these changes may result in long-term clinical and histopathological remission.

FMT is being actively explored as a means of altering the intestinal microbiome for therapeutic benefit in IBD. The rationale supporting FMT as a putative therapeutic modality includes the observation that active IBD is characterized by reduced colonic microbial diversity and the hypothesis that "healthy" donor microbiota may restore homeostasis to the aberrant microbiome and host immune response. Initial interest in utilizing FMT for IBD stemmed from the great success of FMT in treating recurrent or refractory Clostridium difficile infections, another disorder characterized by loss of diversity in the microbiome. ${ }^{101}$ Unfortunately, results of clinical studies exploring FMT as a therapy for IBD have been less robust. ${ }^{69,102,103}$ Although some smaller studies have shown potential efficacy of FMT in UC, two larger randomized placebo control trials of FMT in UC demonstrated little to no efficacy. ${ }^{104-106}$ There are currently insufficient data to support the use of FMT for treatment of CD, but given the small number of studies and small patient sample sizes, additional studies are required to draw definitive conclusions on the efficacy of FMT for induction or maintenance of remission in IBD.

Antibiotics have long been utilized to manage IBD. Primarily, antibiotics have been employed as an adjunctive treatment in specific clinical scenarios, such as perianal and intra-abdominal abscesses, fistula and toxic megacolon. ${ }^{24,26}$ However, certain antibiotics such as ciprofloxacin, metronidazole, and rifaximin with their broad-spectrum antimicrobial coverage have been explored as a primary therapy. In theory, antibiotics may alter the clinical course of IBD by several mechanisms, including: decreasing luminal bacteria concentrations, altering the microbial composition to potentially favor the establishment of beneficial bacteria, and decreasing bacterial tissue invasion. ${ }^{26}$ Clinically, treatment with enteral antibiotics has been shown to reduce intestinal inflammation and has been efficacious in treating mucosal inflammation in $\mathrm{CD}$ and pouchitis. ${ }^{24-26,107}$ However, their use as maintenance therapy in CD is less clear. In a randomized, placebo-controlled, blinded study of 213 adults with active $\mathrm{CD}$, a combination of clarithromycin, rifabutin and clofazimine resulted in short-term improvement in remission, but offered no benefit for prevention of long-term relapse. ${ }^{108}$ A meta-analysis evaluating randomized controlled trials in which antibiotic therapy was compared with placebo for the treatment of IBD demonstrated some benefit of using antibiotics to treat IBD. A total of 10 studies were included in the evaluation of antibiotics in $\mathrm{CD}$ and found a pooled odds ratio (OR) for clinical improvement of 1.35 (95\% confidence interval [CI], 1.16-1.58) for the use of antibiotic therapy compared with placebo. For patients with UC, a total of nine studies included in the analysis demonstrated a pooled OR of 2.17 (95\% CI: 1.54-3.05) in favor of antibiotic therapy. ${ }^{109}$ Despite mounting evidence, additional randomized controlled trials are required to guide decisions regarding the specific clinical roles of antibiotics in the treatment of IBD.

Several studies have evaluated probiotics, both in induction and maintenance of remission in IBD. Probiotics, as a whole, have not demonstrated efficacy in the treatment of $\mathrm{CD}$; however, there may be some role for the use of specific probiotics in the management of mild-to-moderate active UC or recurrent pouchitis after ileoanal anastomosis. ${ }^{110}$ VSL\#3 is a highly-concentrated probiotic preparation that contains eight different types of bacteria within the Lactobacillus, Streptococcus and Bifidobacterium spp. In placebo-controlled trials, VSL\#3 was shown to prevent recurrence of pouchitis. ${ }^{111,112}$ While data exist to support the limited utility of certain probiotics in the treatment of IBD, further studies are required to delineate the anti-inflammatory mechanism of probiotics in IBD. ${ }^{113}$

Prebiotics are compounds that change the structure or metabolome of the intestinal microbiota. Inulin and oligofructose are two prebiotics that have been shown to promote the growth of beneficial Bifidobacterium and Lactobacillus spp. both in humans and in rats. ${ }^{114,115}$ Additionally, cellobiose and rice fiber are dietary-fiber sourced prebiotics that have been shown to reduce pro-infammatory cytokines in experimental models of colitis. ${ }^{116}$ Few studies have examined the beneficial clinical effects of prebiotics in active IBD. Benjamin et al in a randomized placebo-controlled study of adults with active $\mathrm{CD}$ evaluated changes in disease activity index after administration of fructo-oligosaccharides (FOS). Despite some changes to immunoloregulation of dendritic cells observed in those receiving FOS, no significant clinical improvements were seen, nor were there significant changes to the fecal microbiome between the groups at baseline or after the 4-week intervention. ${ }^{117}$

Fermentable fiber, another form of prebiotic, is metabolized by colonic bacteria to short chain fatty acids (SCFA), that is, acetate, propionate and butyrate, which are known to modulate cell proliferation, histone acetylation, gene expression and immune response. ${ }^{118}$ SCFA-producing bacterial strains in Clostridia clusters IV, XIVa and XVII from healthy individuals induce colonic regulatory $\mathrm{T}$ cells (Tregs) differentiation, 
expansion and function. ${ }^{119}$ SCFA produced by Clostridia spp have been found to induce CD4+ T regulatory cells (Treg) and reduce colitis in mouse models. ${ }^{69,120}$ In adults with IBD, fecal samples demonstrated reduced concentrations of butyrate and acetate compared with healthy controls, suggesting that SCFA may play some protective role in the prevention of intestinal inflammation. ${ }^{121}$ In animal models of IBD, supplementation with soluble fiber has been shown to reduce intestinal inflammation by increasing the production of SCFA and altering the intestinal microbiome. ${ }^{122,123}$ In humans, dietary fiber may reduce the risk of flare in patients with $C D$, although additional studies are required to recommend fiber supplementation as an anti-inflammatory therapy in IBD. ${ }^{124}$

\section{Future studies}

Defining the characteristics of "disease" and "health" in intestinal microbiota is only the first step in ascertaining the pathogenesis of IBD. To date, specific profiles of intestinal microbiota have not been beneficial as a diagnostic test or biomarker for IBD; and further studies are required to identify whether aspects of the microbiome may be useful in identifying disease phenotype or predicting response to therapy. ${ }^{11,13,14,69}$ Many earlier studies have been wrought with several challenges in delineating causality when reporting microbial changes associated with IBD. Additionally, robust meta-analysis to draw conclusions have been challenging, given the diversity of microbial sampling and important clinical aspects of disease, including disease phenotype, location of disease, and prior pharmacologic exposures. ${ }^{1}$ Fortunately, with evolving experimental technologies to assist in the functional characterization of the microbiome, there is promise to move beyond defining the phenotypic footprint of the microbiome in IBD to identify how the microbiome contributes to: 1) the onset and propagation of disease, and potentially more significantly, 2) how we may manipulate the microbiome as a future treatment of IBD.

\section{Disclosure}

The authors report no conflicts of interest in this work.

\section{References}

1. Miyoshi J, Chang EB. The gut microbiota and inflammatory bowel diseases. Transl Res. 2017;179:38-48.

2. Loftus EV Jr. Clinical epidemiology of inflammatory bowel disease: incidence, prevalence, and environmental influences. Gastroenterology. 2004;126(6):1504-1517.

3. Molodecky NA, Soon IS, Rabi DM, et al. Increasing incidence and prevalence of the inflammatory bowel diseases with time, based on systematic review. Gastroenterology. 2012;142(1):46-54. e42; quiz e30.
4. Ananthakrishnan AN, Khalili H, Konijeti GG, et al. A prospective study of long-term intake of dietary fiber and risk of Crohn's disease and ulcerative colitis.Gastroenterology. 2013;145(5):970-977.

5. Ponder A, Long MD. A clinical review of recent findings in the epidemiology of inflammatory bowel disease.ClinEpidemiol. 2013;5:237-247.

6. Kostic AD, Xavier RJ, Gevers D. The microbiome in inflammatory bowel disease: current status and the future ahead. Gastroenterology. 2014;146(6):1489-1499.

7. Halme L, Paavola-Sakki P, Turunen U, Lappalainen M, Farkkila M, Kontula K. Family and twin studies in inflammatory bowel disease. World J Gastroenterol. 2006;12(23):3668-3672.

8. Yazdanyar S, Kamstrup PR, Tybjaerg-Hansen A, Nordestgaard BG. Penetrance of NOD2/CARD15 genetic variants in the general population. CMAJ. 2010;182(7):661-665.

9. Hansen JJ, Sartor RB. Therapeutic manipulation of the microbiome in IBD: current results and future approaches. Curr Treat Options Gastroenterol. 2015;13(1):105-120.

10. Frank DN, Robertson CE, Hamm CM, et al. Disease phenotype and genotype are associated with shifts in intestinal-associated microbiota in inflammatory bowel diseases. Inflamm Bowel Dis. 2011; 17(1):179-184.

11. Morgan XC, Tickle TL, Sokol H, et al. Dysfunction of the intestinal microbiome in inflammatory bowel disease and treatment. Genome Biol. 2012;13(9):R79.

12. Li J, Butcher J, Mack D, Stintzi A. Functional impacts of the intestinal microbiome in the pathogenesis of inflammatory bowel disease. Inflamm Bowel Dis. 2015;21(1):139-153.

13. Gevers D, Kugathasan S, Denson LA, et al. The treatment-naive microbiome in new-onset Crohn's disease. Cell Host Microbe. 2014; 15(3):382-392.

14. Frank DN, St Amand AL, Feldman RA, Boedeker EC, Harpaz N, Pace NR. Molecular-phylogenetic characterization of microbial community imbalances in human inflammatory bowel diseases. ProcNatlAcadSci US A. 2007;104(34):13780-13785.

15. Ott SJ, Musfeldt M, Wenderoth DF, et al. Reduction in diversity of the colonic mucosa associated bacterial microflora in patients with active inflammatory bowel disease. Gut. 2004;53(5):685-693.

16. Manichanh C, Rigottier-Gois L, Bonnaud E, et al. Reduced diversity of faecalmicrobiota in Crohn's disease revealed by a metagenomic approach. Gut. 2006;55(2):205-211.

17. Fichera A, McCormack R, Rubin MA, Hurst RD, Michelassi F. Longterm outcome of surgically treated Crohn's colitis: a prospective study. Dis Colon Rectum. 2005;48(5):963-969.

18. Lee YK, Mazmanian SK. Has the microbiota played a critical role in the evolution of the adaptive immune system? Science. 2010;330(6012):1768-1773.

19. Jostins L, Ripke S, Weersma RK, et al. Host-microbe interactions have shaped the genetic architecture of inflammatory bowel disease. Nature. 2012;491(7422):119-124.

20. McGovern DP, Jones MR, Taylor KD, et al; International IBD Genetics Consortium. Fucosyltransferase 2 (FUT2) non-secretor status is associated with Crohn's disease. Hum Mol Genet. 2010;19(17):3468-3476.

21. Rausch P, Rehman A, Kunzel S, et al. Colonic mucosa-associated microbiota is influenced by an interaction of Crohn disease and FUT2 (Secretor) genotype. ProcNatlAcadSci U SA. 2011;108(47):19030-19035.

22. Elinav E, Strowig T, Kau AL, et al. NLRP6 inflammasome regulates colonic microbial ecology and risk for colitis. Cell. 2011;145(5):745-757.

23. Dheer R, Santaolalla R, Davies JM, et al. Intestinal epithelial toll-like receptor 4 signaling affects epithelial function and colonic microbiota and promotes a risk for transmissible colitis. Infect Immun. 2016;84(3):798-810.

24. Sartor RB. Therapeutic manipulation of the enteric microflora in inflammatory bowel diseases: antibiotics, probiotics, and prebiotics. Gastroenterology. 2004;126(6):1620-1633.

25. Rietdijk ST, D'Haens GR.Recent developments in the treatment of inflammatory bowel disease.J Dig Dis. 2013;14(6):282-287. 
26. Perencevich M, Burakoff R. Use of antibiotics in the treatment of inflammatory bowel disease. Inflamm Bowel Dis. 2006;12(7):651-664.

27. Saleh M, Trinchieri G. Innate immune mechanisms of colitis and colitis-associated colorectal cancer. Nat Rev Immunol. 2011;11(1):9-20.

28. Kennedy RJ, Hoper M, Deodhar K, Erwin PJ, Kirk SJ, Gardiner KR. Interleukin 10-deficient colitis: new similarities to human inflammatory bowel disease. Br J Surg. 2000;87(10):1346-1351.

29. Human Microbiome Project C. Structure, function and diversity of the healthy human microbiome. Nature. 2012;486(7402):207-214.

30. Dave M, Higgins PD, Middha S, Rioux KP. The human gut microbiome: current knowledge, challenges, and future directions. Transl Res. 2012;160(4):246-257.

31. Qin J, Li R, Raes J, et al. A human gut microbial gene catalogue established by metagenomic sequencing. Nature. 2010;464(7285):59-65.

32. Eckburg PB, Bik EM, Bernstein CN, et al. Diversity of the human intestinal microbial flora. Science. 2005;308(5728):1635-1638.

33. Claesson MJ, Jeffery IB, Conde S, et al. Gut microbiota composition correlates with diet and health in the elderly. Nature. 2012;488(7410): 178-184.

34. Dominguez-Bello MG, Blaser MJ, Ley RE, Knight R. Development of the human gastrointestinal microbiota and insights from highthroughput sequencing. Gastroenterology. 2011;140(6):1713-1719.

35. Yatsunenko T, Rey FE, Manary MJ, et al. Human gut microbiome viewed across age and geography. Nature. 2012;486(7402):222-227.

36. Lim ES, Zhou Y, Zhao G, et al. Early life dynamics of the human gut virome and bacterial microbiome in infants. Nat Med. 2015;21(10): $1228-1234$

37. Jakobsson HE, Abrahamsson TR, Jenmalm MC, et al. Decreased gut microbiota diversity, delayed Bacteroidetescolonisation and reduced Th1 responses in infants delivered by caesarean section. Gut. 2014;63(4):559-566.

38. Bager P, Simonsen J, Nielsen NM, Frisch M. Cesarean section and offspring's risk of inflammatory bowel disease: a national cohort study. Inflamm Bowel Dis. 2012;18(5):857-862.

39. Bernstein CN, Banerjee A, Targownik LE, et al. Cesarean section delivery is not a risk factor for development of inflammatory bowel disease: apopulation-based analysis. ClinGastroenterolHepatol. 2016;14(1): 50-57.

40. Bezirtzoglou E, Tsiotsias A, Welling GW. Microbiota profile in feces of breast- and formula-fed newborns by using fluorescence in situ hybridization (FISH). Anaerobe. 2011;17(6):478-482.

41. Penders J, Thijs C, Vink C, et al. Factors influencing the composition of the intestinal microbiota in early infancy. Pediatrics. 2006;118(2): 511-521.

42. Praveen P, Jordan F, Priami C, Morine MJ. The role of breast-feeding in infant immune system: a systems perspective on the intestinal microbiome. Microbiome. 2015;3:41

43. Barclay AR, Russell RK, Wilson ML, Gilmour WH, Satsangi J, Wilson DC. Systematic review: the role of breastfeeding in the development of pediatric inflammatory bowel disease. J Pediatr. 2009;155(3):421-426.

44. Backhed F, Roswall J, Peng Y, et al. Dynamics and stabilization of the human gut microbiome during the first year of life. Cell Host Microbe. 2015;17(5):690-703.

45. Planer JD, Peng Y, Kau AL, et al. Development of the gut microbiota and mucosal IgA responses in twins and gnotobiotic mice. Nature. 2016;534(7606):263-266.

46. Lozupone CA, Stombaugh JI, Gordon JI, Jansson JK, Knight R. Diversity, stability and resilience of the human gut microbiota. Nature. 2012;489(7415):220-230.

47. David LA, Maurice CF, Carmody RN, et al. Diet rapidly and reproducibly alters the human gut microbiome. Nature. 2014;505(7484):559-563.

48. Wu GD, Chen J, Hoffmann C, et al. Linking long-term dietary patterns with gut microbial enterotypes. Science. 2011;334(6052):105-108.

49. De Filippo C, Cavalieri D, Di Paola M, et al. Impact of diet in shaping gut microbiota revealed by a comparative study in children from Europe and rural Africa. ProcNatlAcadSci USA. 2010;107(33):14691-14696.
50. Devkota S, Wang Y, Musch MW, et al. Dietary-fat-induced taurocholic acid promotes pathobiont expansion and colitis in Il10-/- mice. Nature. 2012;487(7405):104-108.

51. Ananthakrishnan AN, Khalili H, Higuchi LM, et al. Higher predicted vitamin D status is associated with reduced risk of Crohn's disease. Gastroenterology. 2012;142(3):482-489.

52. Garg M, Rosella O, Lubel JS, Gibson PR. Association of circulating vitamin D concentrations with intestinal but not systemic inflammation in inflammatory bowel disease. Inflamm Bowel Dis. 2013; 19(12):2634-2643.

53. Bashir M, Prietl B, Tauschmann M, et al. Effects of high doses of vita$\min \mathrm{D} 3$ on mucosa-associated gut microbiome vary between regions of the human gastrointestinal tract. Eur J Nutr. 2016;55(4):1479-1489.

54. Lupp C, Robertson ML, Wickham ME, et al. Host-mediated inflammation disrupts the intestinal microbiota and promotes the overgrowth of Enterobacteriaceae. Cell Host Microbe. 2007;2(2):119-129.

55. Darfeuille-Michaud A, Boudeau J, Bulois P, et al. High prevalence of adherent-invasive Escherichia coli associated with ileal mucosa in Crohn's disease. Gastroenterology. 2004;127(2):412-421.

56. Ohkusa T, Sato N, Ogihara T, Morita K, Ogawa M, Okayasu I. Fusobacteriumvarium localized in the colonic mucosa of patients with ulcerative colitis stimulates species-specific antibody. $J$ GastroenterolHepatol. 2002;17(8):849-853.

57. Fite A, Macfarlane S, Furrie E, et al. Longitudinal analyses of gut mucosal microbiotas in ulcerative colitis in relation to patient age and disease severity and duration. J ClinMicrobiol. 2013;51(3): 849-856.

58. Rajilic-Stojanovic M, Shanahan F, Guarner F, de Vos WM. Phylogenetic analysis of dysbiosis in ulcerative colitis during remission. Inflamm Bowel Dis. 2013;19(3):481-488.

59. Ott SJ, Kuhbacher T, Musfeldt M, et al. Fungi and inflammatory bowel diseases: alterations of composition and diversity. Scand J Gastroenterol. 2008;43(7):831-841.

60. Li Q, Wang C, Tang C, He Q, Li N, Li J. Dysbiosis of gut fungal microbiota is associated with mucosal inflammation in Crohn's disease. J ClinGastroenterol. 2014;48(6):513-523.

61. Lewis JD, Chen EZ, Baldassano RN, et al. Inflammation, antibiotics, and diet as environmental stressors of the gut microbiome in pediatric Crohn'sdisease. Cell Host Microbe. 2015;18(4):489-500.

62. Hoarau G, Mukherjee PK, Gower-Rousseau C, et al. Bacteriome and Mycobiome Interactions Underscore Microbial Dysbiosis in Familial Crohn's Disease. MBio. 2016;7(5).pii: e01250-16.

63. Lepage P, Colombet J, Marteau P, Sime-Ngando T, Doré J, Leclerc M. Dysbiosis in inflammatory bowel disease: a role for bacteriophages? Gut. 2008;57(3):424-425.

64. Norman JM, Handley SA, Baldridge MT, et al. Disease-specific alterations in the enteric virome in inflammatory bowel disease. Cell. 2015;160(3):447-460.

65. Sepehri S, Kotlowski R, Bernstein CN, Krause DO. Microbial diversity of inflamed and noninflamed gut biopsy tissues in inflammatory bowel disease. Inflamm Bowel Dis. 2007;13(6):675-683.

66. Ogura Y, Bonen DK, Inohara N, et al. A frameshift mutation in NOD2 associated with susceptibility to Crohn's disease. Nature. 2001; 411(6837):603-606.

67. Philpott DJ, Girardin SE. Crohn's disease-associated Nod2 mutants reduce IL10 transcription. Nat Immunol. 2009;10(5):455-457.

68. Petnicki-Ocwieja T, Hrncir T, Liu YJ, et al. Nod2 is required for the regulation of commensal microbiota in the intestine. ProcNatlAcadSci US A. 2009;106(37):15813-15818.

69. Xavier RJ. Microbiota as therapeutic targets. Dig Dis. 2016;34(5): 558-565.

70. Iliev ID, Funari VA, Taylor KD, et al. Interactions between commensal fungi and the C-type lectin receptor Dectin-1 influence colitis. Science. 2012;336(6086):1314-1317.

71. Hviid A, Svanstrom H, Frisch M. Antibiotic use and inflammatory bowel diseases in childhood. Gut. 2011;60(1):49-54. 
72. Virta L, Auvinen A, Helenius H, Huovinen P, Kolho KL. Association of repeated exposure to antibiotics with the development of pediatric Crohn's disease-a nationwide, register-based finnish case-control study. Am J Epidemiol. 2012;175(8):775-784.

73. Kronman MP, Zaoutis TE, Haynes K, Feng R, Coffin SE. Antibiotic exposure and IBD development among children: a population-based cohort study. Pediatrics. 2012;130(4):e794-e803.

74. Ungaro R, Bernstein CN, Gearry R, et al. Antibiotics associated with increased risk of new-onset Crohn's disease but not ulcerative colitis: a meta-analysis. Am J Gastroenterol. 2014;109(11):1728-1738.

75. Investigators IBDiES, Tjonneland $\mathrm{A}$, Overvad $\mathrm{K}$, et al. Linoleic acid, a dietary n-6 polyunsaturated fatty acid, and the aetiology of ulcerative colitis: a nested case-control study within a European prospective cohort study. Gut. 2009;58(12):1606-1611.

76. Ananthakrishnan AN, Khalili H, Konijeti GG, et al. Long-term intake of dietary fat and risk of ulcerative colitis and Crohn's disease. Gut. 2014;63(5):776-784

77. Ghosh S, DeCoffe D, Brown K, et al. Fish oil attenuates omega-6 polyunsaturated fatty acid-induced dysbiosis and infectious colitis but impairs LPS dephosphorylation activity causing sepsis. PLoS One. 2013;8(2):e55468.

78. Gerasimidis K, Bertz M, Hanske L, et al. Decline in presumptively protective gut bacterial species and metabolites are paradoxically associated with disease improvement in pediatric Crohn's disease during enteral nutrition. Inflamm Bowel Dis. 2014;20(5):861-871.

79. Sokol H, Seksik P, Furet JP, et al. Low counts of Faecalibacteriumprausnitzii in colitis microbiota. Inflamm Bowel Dis. 2009;15(8):1183-1189.

80. Lewis JD, Abreu MT. Diet as a trigger or therapy for inflammatory bowel diseases. Gastroenterology. 2017;152(2):398-414.e6.

81. Borrelli O, Cordischi L, Cirulli M, et al. Polymeric diet alone versus corticosteroids in the treatment of active pediatric Crohn's disease: a randomized controlled open-label trial. ClinGastroenterolHepatol. 2006;4(6):744-753.

82. Takagi S, Utsunomiya K, Kuriyama S, et al. Effectiveness of an 'half elemental diet' as maintenance therapy for Crohn's disease: a randomized-controlled trial. Aliment PharmacolTher. 2006;24(9):1333-1340.

83. Grover Z, Muir R, Lewindon P. Exclusive enteral nutrition induces early clinical, mucosal and transmural remission in paediatric Crohn's disease. J Gastroenterol. 2014;49(4):638-645.

84. Lee D, Baldassano RN, Otley AR, et al. Comparative effectiveness of nutritional and biological therapy in North American Children with active Crohn'sdisease. Inflamm Bowel Dis. 2015;21(8): 1786-1793.

85. Suskind DL, Wahbeh G, Cohen SA, et al. Patients perceive clinical benefit with the specific carbohydrate diet for inflammatory bowel disease. Dig Dis Sci. 2016;61(11):3255-3260.

86. Suskind DL, Wahbeh G, Gregory N, Vendettuoli H, Christie D. Nutritional therapy in pediatric Crohn disease: the specific carbohydrate diet. J PediatrGastroenterolNutr. 2014;58(1):87-91.

87. Cohen SA, Gold BD, Oliva S, et al. Clinical and mucosal improvement with specific carbohydrate diet in pediatric Crohn disease. J PediatrGastroenterolNutr. 2014;59(4):516-521.

88. Obih C, Wahbeh G, Lee D, et al. Specific carbohydrate diet for pediatric inflammatory bowel disease in clinical practice within an academic IBD center. Nutrition. 2016;32(4):418-425.

89. Kakodkar S, Farooqui AJ, Mikolaitis SL, Mutlu EA. The specific carbohydrate diet for inflammatory bowel disease: a case series. J AcadNutr Diet. 2015;115(8):1226-1232.

90. Suskind DL, Cohen SA, Brittnacher MJ, et al. Clinical and fecal microbial changes with diet therapy in active inflammatory bowel disease. J ClinGastroenterol. Epub2016 Dec 27.

91. Sigall-Boneh R, Pfeffer-Gik T, Segal I, Zangen T, Boaz M, Levine A. Partial enteral nutrition with a Crohn's disease exclusion diet is effective for induction of remission in children and young adults with Crohn's disease. Inflamm Bowel Dis. 2014;20(8):1353-1360.
92. Riordan AM, Hunter JO, Cowan RE, et al. Treatment of active Crohn's disease by exclusion diet: East Anglian multicentre controlled trial. Lancet. 1993;342(8880):1131-1134.

93. Rajendran N, Kumar D. Food-specific IgG4-guided exclusion diets improve symptoms in Crohn's disease: a pilot study. Colorectal Dis. 2011;13(9):1009-1013.

94. Chiba M, Abe T, Tsuda H, et al. Lifestyle-related disease in Crohn's disease: relapse prevention by a semi-vegetarian diet. World J Gastroenterol. 2010;16(20):2484-2495.

95. Gearry RB, Irving PM, Barrett JS, Nathan DM, Shepherd SJ, Gibson PR. Reduction of dietary poorly absorbed short-chain carbohydrates (FODMAPs) improves abdominal symptoms in patients with inflammatory bowel disease-a pilot study. J Crohns Colitis. 2009;3(1): $8-14$.

96. Prince AC, Myers CE, Joyce T, Irving P, Lomer M, Whelan K. Fermentable carbohydrate restriction (Low FODMAP Diet) in clinical practice improves functional gastrointestinal symptoms in patients with inflammatory bowel disease. Inflamm Bowel Dis. 2016;22(5):1129-1136.

97. Halmos EP, Christophersen CT, Bird AR, Shepherd SJ, Muir JG, Gibson PR. Consistent prebiotic effect on gut microbiotawith altered FODMAP intake in patients with Crohn'sdisease: a randomised, controlled cross-over trial of well-defined diets. ClinTranslGastroenterol. 2016;7:e164.

98. Zimmer J, Lange B, Frick JS, et al. A vegan or vegetarian diet substantially alters the human colonic faecal microbiota. Eur J ClinNutr. 2012;66(1):53-60.

99. Chassaing B, Koren O, Goodrich JK, et al. Dietary emulsifiers impact the mouse gut microbiota promoting colitis and metabolic syndrome. Nature. 2015;519(7541):92-96.

100. Nickerson KP, Chanin R, McDonald C. Deregulation of intestinal antimicrobial defense by the dietary additive, maltodextrin. Gut Microbes. 2015;6(1):78-83.

101. van Nood E, Vrieze A, Nieuwdorp M, et al. Duodenal infusion of donor feces for recurrent Clostridium difficile. N Engl J Med. 2013; 368(5):407-415.

102. Ianiro G, Bibbo S, Scaldaferri F, Gasbarrini A, Cammarota G. Fecal microbiota transplantation in inflammatory bowel disease: beyond the excitement. Medicine (Baltimore). 2014;93(19):e97.

103. Kump PK, Grochenig HP, Lackner S, et al. Alteration of intestinal dysbiosis by fecal microbiota transplantation does not induce remission in patients with chronic active ulcerative colitis. Inflamm Bowel Dis. 2013;19(10):2155-2165.

104. Kunde S, Pham A, Bonczyk S, et al. Safety, tolerability, and clinical response after fecal transplantation in children and young adults with ulcerative colitis. J PediatrGastroenterolNutr. 2013;56(6): 597-601.

105. Moayyedi P, Surette MG, Kim PT, et al. Fecal microbiota transplantation induces remission in patients with active ulcerative colitis in a randomized controlled trial. Gastroenterology. 2015;149(1): $102-109$ e6

106. Rossen NG, Fuentes S, van der Spek MJ, et al. Findings from a randomized controlled trial of fecal transplantation for patients with ulcerative colitis. Gastroenterology. 2015;149(1):110-118.e4.

107. Casellas F, Borruel N, Papo M, et al. Antiinflammatory effects of enterically coated amoxicillin-clavulanic acid in active ulcerative colitis. Inflamm Bowel Dis. 1998;4(1):1-5.

108. Selby W, Pavli P, Crotty B, et al; Antibiotics in Crohn's Disease Study Group. Two-year combination antibiotic therapy with clarithromycin, rifabutin, and clofazimine for Crohn's disease. Gastroenterology. 2007;132(7):2313-2319.

109. Wang SL, Wang ZR, Yang CQ. Meta-analysis of broad-spectrum antibiotic therapy in patients with active inflammatory bowel disease. ExpTher Med. 2012;4(6):1051-1056.

110. Mack DR. Probiotics in inflammatory bowel diseases and associated conditions. Nutrients. 2011;3(2):245-264. 
111. Gionchetti P, Rizzello F, Venturi A, et al. Oral bacteriotherapy as maintenance treatment in patients with chronic pouchitis: a double-blind, placebo-controlled trial. Gastroenterology. 2000;119(2):305-309.

112. Mimura T, Rizzello F, Helwig U, et al. Once daily high dose probiotic therapy (VSL\#3) for maintaining remission in recurrent or refractory pouchitis.Gut. 2004;53(1):108-114.

113. Matsuoka K, Kanai T. The gut microbiota and inflammatory bowel disease. SeminImmunopathol. 2015;37(1):47-55.

114. Guarner F. Prebiotics in inflammatory bowel diseases. Br J Nutr. 2007;98(Suppl 1):S85-S89.

115. Arribas B, Suarez-Pereira E, Ortiz Mellet C, et al. Di-D-fructose dianhydride-enriched caramels: effect on colon microbiota, inflammation, and tissue damage in trinitrobenzenesulfonic acid-induced colitic rats. J Agric Food Chem. 2010;58(10):6476-6484.

116. Nishimura T, Andoh A, Hashimoto T, Kobori A, Tsujikawa T, Fujiyama Y. Cellobiose prevents the development of dextran sulfate sodium (DSS)-induced experimental colitis. J ClinBiochemNutr. 2010;46(2):105-110.

117. Benjamin JL, Hedin CR, Koutsoumpas A, et al. Randomised, doubleblind, placebo-controlled trial of fructo-oligosaccharides in active Crohn's disease. Gut. 2011;60(7):923-929.
118. Kim S, Kim JH, Park BO, KwakYS. Perspectives on the therapeutic potential of short-chain fatty acid receptors. BMB Rep. 2014;47(3):173-178.

119. Atarashi K, Tanoue T, Shima T, et al. Induction of colonic regulatory T cells by indigenous Clostridium species. Science. 2011;331(6015):337-341.

120. Smith PM, Howitt MR, Panikov N, et al. The microbial metabolites, short-chain fatty acids, regulate colonic Treg cell homeostasis. Science. 2013;341(6145):569-573.

121. Huda-Faujan N, Abdulamir AS, Fatimah AB, et al. The impact of the level of the intestinal short chain fatty acids in inflammatory bowel disease patients versus healthy subjects. Open Biochem J. 2010;4:53-58.

122. Koleva PT, Valcheva RS, Sun X, Gänzle MG, Dieleman LA. Inulin and fructo-oligosaccharides have divergent effects on colitis and commensal microbiota in HLA-B27 transgenic rats. Br J Nutr. 2012; 108(9):1633-1643.

123. Joo E, Yamane S, Hamasaki A, et al. Enteral supplement enriched with glutamine, fiber, and oligosaccharide attenuates experimental colitis in mice. Nutrition. 2013;29(3):549-555.

124. Brotherton CS, Martin CA, Long MD, Kappelman MD, Sandler RS Avoidance of fiber is associated with greater risk of Crohn's disease flare in a 6-month period. Clin Gastroenterol Hepatol. 2016;14(8): 1130-1136.
Journal of Inflammation Research

\section{Publish your work in this journal}

The Journal of Inflammation Research is an international, peer-reviewed open access journal that welcomes laboratory and clinical findings on the molecular basis, cell biology and pharmacology of inflammation including original research, reviews, symposium reports, hypothesis formation and commentaries on: acute/chronic inflammation; mediators of

\section{Dovepress}

inflammation; cellular processes; molecular mechanisms; pharmacology and novel anti-inflammatory drugs; clinical conditions involving inflammation. The manuscript management system is completely online and includes a very quick and fair peer-review system. Visit http://www.dove press.com/testimonials.php to read real quotes from published authors. 